\title{
Orbital nodal surfaces: Topological challenges for density functionals
}

\author{
Thilo Aschebrock, ${ }^{1}$ Rickard Armiento, ${ }^{2}$ and Stephan Kümmel ${ }^{1}$ \\ ${ }^{1}$ Theoretical Physics IV, University of Bayreuth, D-95440 Bayreuth, Germany \\ ${ }^{2}$ Department of Physics, Chemistry and Biology (IFM), Linköping University, SE-58183 Linköping, Sweden
}

(Received 22 December 2016; published 15 June 2017)

\begin{abstract}
Nodal surfaces of orbitals, in particular of the highest occupied one, play a special role in Kohn-Sham density-functional theory. The exact Kohn-Sham exchange potential, for example, shows a protruding ridge along such nodal surfaces, leading to the counterintuitive feature of a potential that goes to different asymptotic limits in different directions. We show here that nodal surfaces can heavily affect the potential of semilocal densityfunctional approximations. For the functional derivatives of the Armiento-Kümmel (AK13) [Phys. Rev. Lett. 111, 036402 (2013)] and Becke88 [Phys. Rev. A 38, 3098 (1988)] energy functionals, i.e., the corresponding semilocal exchange potentials, as well as the Becke-Johnson [J. Chem. Phys. 124, 221101 (2006)] and van LeeuwenBaerends (LB94) [Phys. Rev. A 49, 2421 (1994)] model potentials, we explicitly demonstrate exponential divergences in the vicinity of nodal surfaces. We further point out that many other semilocal potentials have similar features. Such divergences pose a challenge for the convergence of numerical solutions of the Kohn-Sham equations. We prove that for exchange functionals of the generalized gradient approximation (GGA) form, enforcing correct asymptotic behavior of the potential or energy density necessarily leads to irregular behavior on or near orbital nodal surfaces. We formulate constraints on the GGA exchange enhancement factor for avoiding such divergences.
\end{abstract}

DOI: 10.1103/PhysRevB.95.245118

\section{INTRODUCTION}

Kohn-Sham (KS) density-functional theory (DFT) [1,2] has become the method of choice for calculating the electronic structure of physical, chemical, and biological systems. This success is based on the favorable ratio of accuracy to computational cost that DFT offers, especially with semilocal approximations for the exchange-correlation (xc) energy $E_{\mathrm{xc}}[n(\mathbf{r})]$. However, while the low computational cost of semilocal functionals has very much contributed to the success of DFT because it enables access to large systems of practical relevance, the functional derivatives of typical semilocal functionals, i.e., their corresponding xc potentials, miss important features of the exact xc potential, in particular discontinuities $[3,4]$ and step structures [5-9] that are relevant, e.g., in charge-transfer situations [10-12] and ionization processes [5,13-16]. Many attempts have been made to incorporate some of the missing features into semilocal DFT [17-27]. In recent years, it was the Becke-Johnson (BJ) model potential [28] in particular that sparked interest in this respect [22,29-35]. Its key characteristic is to effectively mimic nonlocal exchange features in the asymptotic behavior of the potential by means of having a nonzero limiting value far away from a finite system. This key characteristic was later adopted for the Armiento-Kümmel 2013 energy functional (AK13) by two of the present authors [36]. While this asymptotic behavior of the $\mathrm{xc}$ potential has a variety of implications [37], one particularly striking consequence becomes most apparent in systems with nodal surfaces of the highest occupied (homo) KS orbital.

Such orbital nodal surfaces have emerged as a topic of particular interest in DFT in recent years. To be precise, by the term "nodal surface" we refer here to the situation in which the highest occupied KS orbital in the ground state of a finite system has a nodal surface that extends to infinity. The first observation that such nodal surfaces of the homo play a special role in KS theory came from studying the exact KS exchange potential. It has been shown-first in the localized Hartree-Fock approximation [38,39] and then exactly via optimized effective potential (OEP) calculations [40,41] - that a pronounced "ridge" appears in the bare exchange potential along a nodal surface at intermediate distances. At large distances, it contracts exponentially to a set of zero measure. As visualization of this counterintuitive feature might be helpful for further discussion, we refer to Fig. 3 of Ref. [41] and to Fig. 3 of this article. It has been argued that such ridges are of practical relevance as they can significantly affect unoccupied KS orbitals and eigenvalues [38,39], i.e., quantities that are important in particular for time-dependent DFT calculations or perturbation theory methods.

However, the ridge feature has also sparked interest from a fundamental perspective. Along a ridge, the exact KS exchange potential asymptotically goes to a nonzero constant. In all other directions of space, it asymptotically falls off to zero. One may argue that no physical potential may go to different asymptotic limits in different directions of space, because this would allow us to build some sort of perpetuum mobile: Consider an electron from the center of the system out to infinity along some direction that does not coincide with the nodal surface, i.e., does not coincide with the ridge. Common sense tells us that independent of the shape of the potential, there cannot be any interaction between the electron and the system when the electron is at infinity, so at infinity the electron can be moved at zero energy cost to any point, e.g., a point on the nodal surface and thus to the top of the ridge. Then bring the electron back into the center of the system right along the ridge. When the full cycle has been completed, the electron will have gained an amount of energy that is proportional to the height of the ridge "out of nothing." Obviously this cannot be, so how can the KS exact exchange potential show such an "unphysical" feature? The gist of the matter is that the KS potential is not a physical potential, but a mathematical object defined as a functional derivative [46]. 


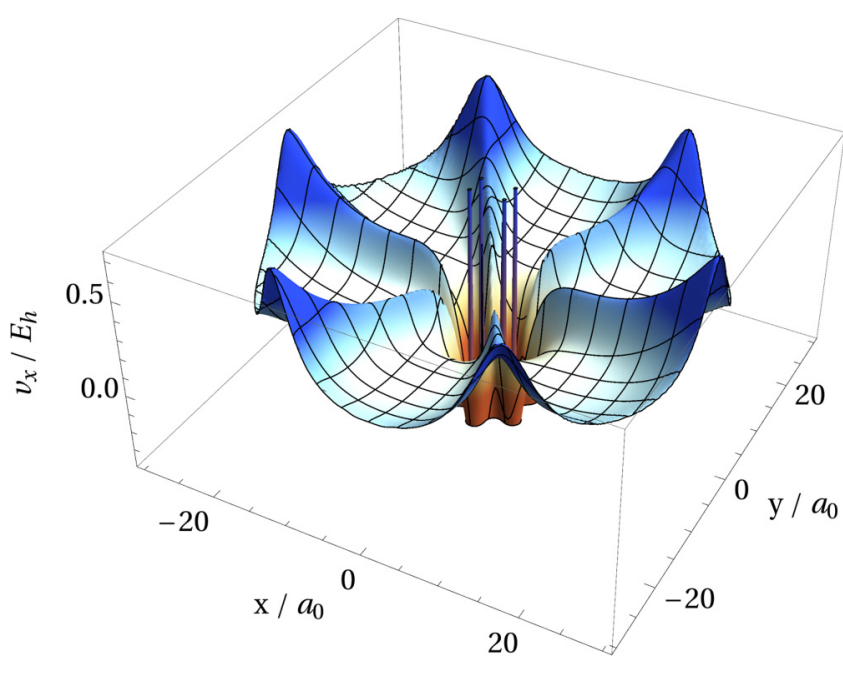

FIG. 1. Contour plot of the AK13 potential landscape of benzene in the plane of the molecule. The semilocal AK13 potential diverges exponentially along the three nodal planes. Due to serious numerical difficulties, which are intensified by the nodal surfaces issues, the AK13 potential could not be calculated self-consistently. For this plot, the AK13 potential was evaluated on a tightly converged selfconsistent LDA valence density obtained from the Bayreuth version [42] of the PARSEC real-space code [43] with a sphere radius of $30 a_{0}$ and a grid spacing of $0.3 a_{0}$.

Therefore, one cannot move electrons (KS particles are not electrons) around in the KS potential and obviously cannot build an "exact exchange perpetuum mobile." Nevertheless, the question of whether the nonvanishing asymptotic constants and associated ridge structures are only a feature of bare exchange, or whether these signatures of nodal surfaces would prevail also in the total exchange-correlation potential, has been debated $[47,48]$. The discussion of nodal surfaces has gained further momentum through the recent discovery [49] that nodal surfaces can also lessen the significance of so-called "iso-orbital indicator" functionals that have been frequently

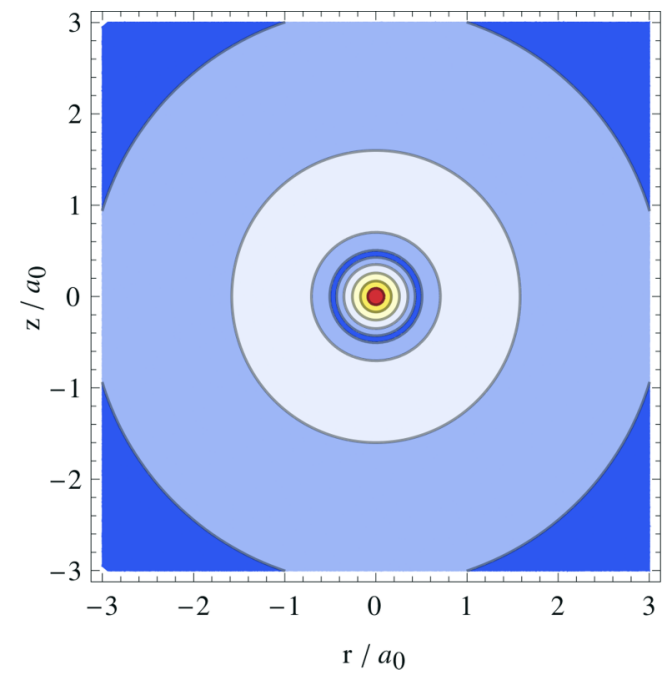

used, e.g., for the purpose of eliminating self-correlation errors.

One may wonder why nodal surfaces of an orbital can play such a special role in DFT, although a ground-state density itself does not have nodes [50]. The answer is that although the ground-state density is nodeless, it is nevertheless strongly affected by homo nodal surfaces. While the asymptotic density is governed by the homo in almost all of space, we discuss in this work that not all asymptotic properties of the density (such as certain partial derivatives) are determined solely by the homo in the vicinity of nodal surfaces. As a consequence, semilocal functionals that use derivative information can show unexpected and quite violent features in the vicinity of nodal surfaces. This is exemplified by Fig. 1, which shows the AK13 potential for benzene: The potential diverges exponentially in the vicinity of a nodal surface. We show in this paper that such an anomalous behavior is also found for the BJ exchange potential and even-though in somewhat weaker form - also for generalized gradient approximations (GGAs) with less strongly diverging enhancement factors than that of AK13. The most prominent example of such an affected GGA is the Becke 1988 exchange functional (B88) [51], which is the semilocal ingredient of the hybrid functional B3LYP [52,53]. As the latter is one of the most used density functionals for molecular systems, the relevance of the nodal surface features that we discuss here is apparent.

In addition to conceptual questions that a diverging potential raises, divergences at nodal surfaces can also severely hinder self-consistent calculations. This has far reaching consequences, because many systems of practical relevance, in particular organic molecules from a chemical and biological context, exhibit at least one and often even multiple nodal planes. Furthermore, simple systems can also show nodal planes. As a prime example, Fig. 2 visualizes the orbital structure of the boron atom with its noded $p_{z}$ orbital. Similar features are seen in the density of many open-shell atoms. In first-principles electronic structure theory for finite systems, the occurrence of nodal surfaces is therefore the rule rather than the exception.

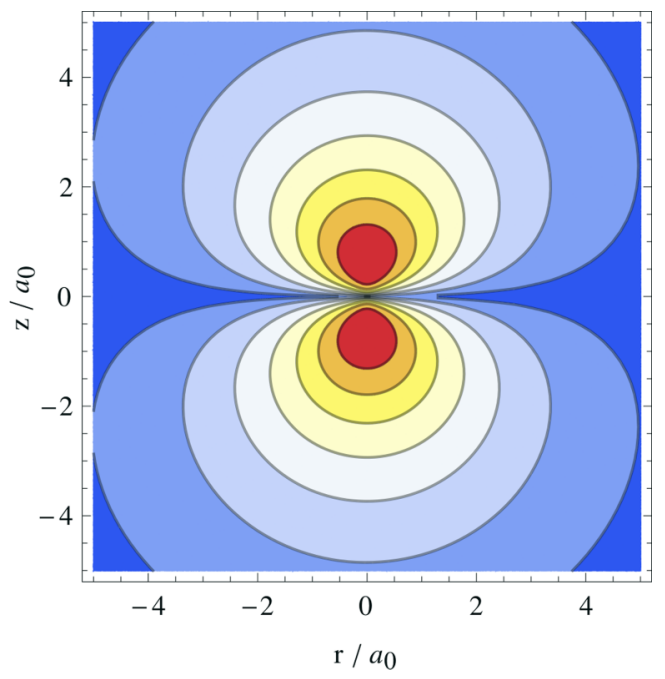

FIG. 2. Contour plots of the highest (right) and of the second highest occupied spin-up orbital densities (left) for the boron atom based on self-consistent LSDA all-electron calculations with the real-space grid program DARSEC [44,45]. While the homo-1 is of perfect spherical symmetry and exhibits a single radial node, the homo is a paradigm of a $p_{z}$ orbital with its nodal plane at $z=0$. 
In this paper, we take a close look at the density in the vicinity of nodal surfaces, and we show how nodal surfaces can affect density functionals. Toward that end, we first introduce a minimal model of auxiliary orbitals that serves as a paradigm system with nodal planes. The model is then utilized to investigate the asymptotic behavior of the density, reduced density derivatives, and the kinetic energy density in the vicinity of nodal surfaces. Based on these findings, we study the exchange potentials of AK13 and B88, as well as the model potential of BJ and van Leeuwen and Baerends (LB94) [17], and we comment on several other functionals. We deliberately focus only on the exchange part in this analysis of approximate functionals, as the design criteria that led to irregular behavior in the vicinity of asymptotic nodal surfaces are specific to exchange. This is a consequence of the fact that the long-range part of the exact xc potential is dominated by exchange. Consequently, also approximate correlation potentials are typically constructed such that they vanish considerably faster than their exchange counterparts in the asymptotic region. Therefore, they are of little relevance in the present context. We conclude by discussing the implications of our findings. Hartree atomic units are used throughout.

\section{MINIMAL NODAL SURFACE MODEL}

In the following, we introduce a minimal model describing the simplest kind of nodal plane. The model is of general utility and inspired by the ground state of a neutral cluster of four sodium atoms [41]. It can be motivated equally well as a schematic variant of the boron-atom shown in Fig. 2. When the $\mathrm{Na}_{4}$ cluster is described in the pseudopotential approximation [54,55], its two valence orbitals that are each occupied by two electrons are smooth and can be approximated by $s$ and $p$ orbitals, respectively. The energetically lower orbital is of $s$ character, and for simplicity modeled by a $1 s$ orbital. The highest occupied orbital is of $p$ character and chosen to be described by a $2 p_{z}$ orbital. Therefore, the nodal plane in our minimal model is given by the $x-y$ plane and extends to infinity. Due to rotational symmetry with respect to the $z$ axis, we chose cylindrical coordinates $\{r, z, \phi\}$. The density of the minimal model is thus given by

$$
\begin{aligned}
n(r, z)= & 2 n_{s}^{0} \exp \left(-\alpha_{s} \sqrt{r^{2}+z^{2}}\right) \\
& +2 n_{p}^{0} z^{2} \exp \left(-\alpha_{p} \sqrt{r^{2}+z^{2}}\right),
\end{aligned}
$$

where $n_{s}^{0}=\alpha_{s}^{3} / 8 \pi$ and $n_{p}^{0}=\alpha_{p}^{5} / 32 \pi$ for normalization. It is known that the exponential decay lengths $\alpha_{s}$ and $\alpha_{p}$ of the corresponding orbital contributions to the density are determined by their respective eigenvalues. All subsequent results are derived without explicit values for $\alpha_{s}$ and $\alpha_{p}$, given that $\alpha_{s}>\alpha_{p}>0$. Nonetheless, for the purpose of visualization we choose these free parameters inspired by the EXX eigenvalues of the cluster $\mathrm{Na}_{4}$, given in Ref. [41], via $\alpha_{s}=2 \sqrt{-2 \varepsilon_{s}} \approx 1.1873$ and $\alpha_{p}=2 \sqrt{-2 \varepsilon_{p}} \approx 1.0587$, and thus we conclude our model.

The density is asymptotically dominated by the $p_{z}$ orbital except in the neighborhood of the nodal plane $(z=0)$. In fact, for every distance to the nodal plane, $z>0$, there exists a finite distance from the center of the molecule $r$ such that $n(r, z)$ is

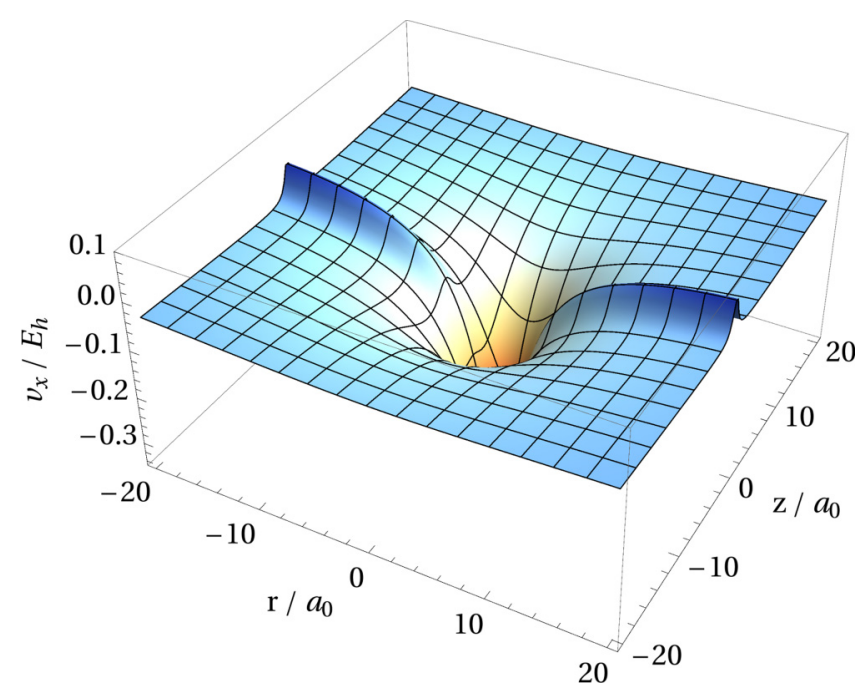

FIG. 3. EXX potential (shown in the KLI approximation [56]) evaluated in the minimal model of a nodal plane located at $z=0$ using MATHEMATICA [57]. The characteristic pronounced "ridge" is visible in the potential along the nodal surface at intermediate distances. For large distances, the ridge contracts exponentially to the nodal plane-a set of zero measure.

arbitrarily accurately described by only its $p$ component,

$$
n_{p}(r, z)=2 n_{p}^{0} z^{2} \exp \left(-\alpha_{p} \sqrt{r^{2}+z^{2}}\right) .
$$

This non-ground-state density, $n_{p}(r, z)$, will be referred to as the pure model of the nodal plane, as it features an actual node. In the limit $r \rightarrow \infty$ and except for a set of zero measure, given by the nodal plane itself, semilocal potentials are completely determined by this density of the pure model.

However, when considering a given finite value of $r$, the $s$-density part of the minimal model contributes noticeably in a small region of space that encloses the nodal plane. We will refer to this region as the transition region, as semilocal quantities in this region are typically determined by the interplay of contributions from both orbitals. In the limit $r \rightarrow$ $\infty$, it contracts exponentially to a set of zero measure. Closely connected to this region is the behavior exactly on the nodal plane. While the $p$ contribution to the density itself vanishes by construction on or along this plane, the $p$ contribution to the Laplacian of the density, $\nabla^{2} n$, remains finite and even dominates in the large- $r$ limit, essentially explaining why some semilocal potentials with critical asymptotics diverge along nodal surfaces, as we will show below.

To showcase the capabilities of this minimal model of a nodal plane, we have plotted the EXX potential evaluated within this model in Fig. 3. For this purpose, the EXX potential is approximated by solving the KLI equation [56] for the fixed auxiliary orbitals of the minimal model. The clearly visible pronounced potential ridge along the nodal plane serves as verification of the model. The behavior of the EXX potential in this figure will be utilized as a reference to the subsequent study of the exchange potentials of AK13, BJ, B88, and LB94. 


\section{RESULTS FOR THE ASYMPTOTICS OF SEMILOCAL FUNCTIONAL EXPRESSIONS}

Before examining the full expressions of semilocal potentials, we will use the minimal model to study the asymptotics of a few semilocal ingredients appearing in such potentials. We begin from the common definitions of reduced spatial derivatives of the density,

$$
s=\frac{|\nabla n|}{2 \gamma n^{4 / 3}}, \quad t=\frac{\nabla^{2} n}{4 \gamma^{2} n^{5 / 3}}, \quad u=\frac{\nabla n \cdot \nabla|\nabla n|}{8 \gamma^{3} n^{3}},
$$

where $\gamma=\left(3 \pi^{2}\right)^{1 / 3}$, and the positive-defined kinetic energy density is

$$
\tau=\frac{1}{2} \sum_{i} f_{i}\left|\nabla \varphi_{i}\right|^{2}
$$

with $f_{i}$ the occupation number of KS orbital $i$.

For an electron density $n(\mathbf{r})$ that decays regularly, i.e., governed by the highest occupied orbital in a spherical symmetric manner, one finds [58]

$$
n(\mathbf{r}) \sim n_{0}|\mathbf{r}|^{q} \exp (-\alpha|\mathbf{r}|) \quad \text { as } \quad|\mathbf{r}| \rightarrow \infty,
$$

where $n_{0}$ and $q$ are system-dependent constants, and the decay parameter

$$
\alpha=2 \sqrt{-2\left(\varepsilon_{\mathrm{ho}}-v_{\mathrm{xc}}^{\infty}\right)}
$$

is determined by the homo eigenvalue $\varepsilon_{\text {ho }}$ relative to the limiting value of the potential $v_{\mathrm{xc}}^{\infty}=\lim _{|\mathbf{r}| \rightarrow \infty} v_{\mathrm{xc}}(\mathbf{r})$.

For a density that decays isotropically according to Eq. (5), one finds that the following characteristic combinations of semilocal components approach nonzero limiting values:

$$
\begin{aligned}
\frac{u}{s^{3}} & \sim 1, \quad \frac{t}{s^{2}} \sim 1-\frac{2}{3} \frac{1}{\ln (s)}, \\
2 \gamma n^{1 / 3} s & \sim \alpha, \quad \frac{\tau}{n} \sim \frac{\alpha^{2}}{8}
\end{aligned}
$$

as $|\mathbf{r}| \rightarrow \infty$.

However, when the density does not decay regularly, e.g., due to a nodal plane of the homo, the asymptotic behaviors of the quantities in Eq. (7) are different. Figures 4-7 show these quantities evaluated in the minimal model via cross sections perpendicular to the nodal plane for several distances from the molecular center $r$.

The behavior of $t / s^{2}=n \nabla^{2} n /|\nabla n|^{2}$ is shown in Fig. 4: Well outside of the nodal plane (for large $z$ values) the semilocal ratio approximately approaches its spherical asymptotic limit, $t / s^{2} \sim 1$. Exactly on the plane, $t / s^{2}$ fails to balance the exponential decay of the density, due to the finite $p$ contribution to $\nabla^{2} n$, and it diverges exponentially as $r \rightarrow \infty$. However, the transition region surrounding this divergence contracts to a set of zero measure in the same limit, leaving the behavior of the quantity $t / s^{2}$ given by the pure model of the nodal plane [cf. Eq. (2)], which can by summarized by the asymptotic relation

$$
\frac{t}{s^{2}} \sim \frac{1}{2}+\left(\frac{1}{8}-\frac{1}{\alpha_{p} r}\right) \alpha_{p}^{2} z^{2}
$$

as $z \rightarrow 0^{+}$. In summary, the quantity $t / s^{2}$ diverges exponentially along a nodal plane, but the region affected by the

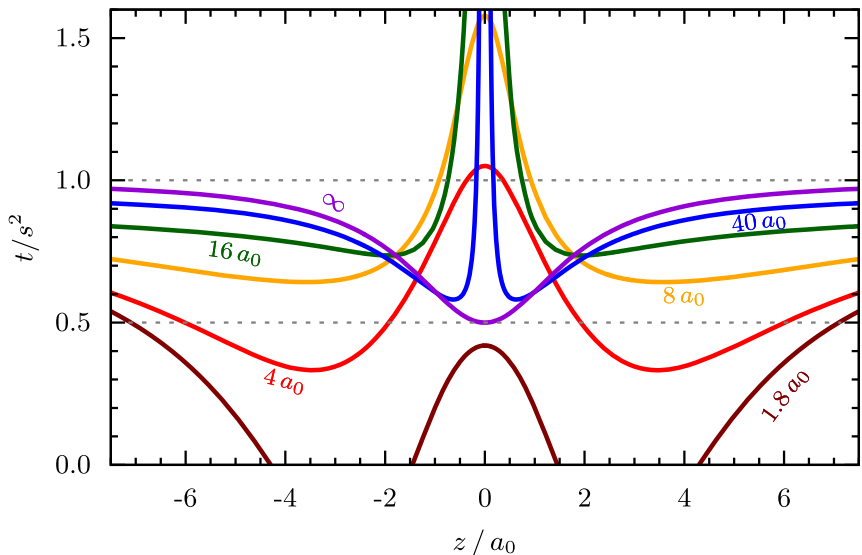

FIG. 4. Cross section of $t / s^{2}$ evaluated in the minimal model of a nodal plane $(z=0)$, cf. Eq. (1). Different lines correspond to different values of $r$ as indicated by the subscripts.

divergence contracts to a set of zero measure leaving an almost everywhere finite limiting value. This value, however, differs considerably from its spherical symmetric limit in the vicinity of the nodal plane.

The ratio $u / s^{3}=n \nabla n \cdot \nabla|\nabla n| /|\nabla n|^{3}$, shown in Fig. 5, behaves similar to $t / s^{2}$ : In the pure model, which is approached as $r \rightarrow \infty$ nearly everywhere, one obtains

$$
\frac{u}{s^{3}} \sim \frac{1}{2}+\left(\frac{1}{4}-\frac{3}{4 \alpha_{p} r}\right) \alpha_{p}^{2} z^{2}
$$

as $z \rightarrow 0^{+}$, and likewise far outside the nodal plane $u / s^{3}$ approaches its ordinary spherical asymptotic limit, $u / s^{3} \sim 1$. However, exactly on the nodal plane this ratio is given solely by the $s$ contribution, thus $u / s^{3}$ converges to the spherical asymptotic limit instead of diverging. This limit is surrounded by a transition region, which features exponentially diverging elements but contracts to the nodal plane as $r \rightarrow \infty$.

So far all diverging contributions to semilocal potentials that we have examined contract to a set of zero measure in the limit $r \rightarrow \infty$, i.e., when evaluated in the pure model of the nodal plane. The quantity $2 \gamma n^{1 / 3} s=|\nabla n| / n$, which is key to the construction of AK13, is different in this respect, as Fig. 6

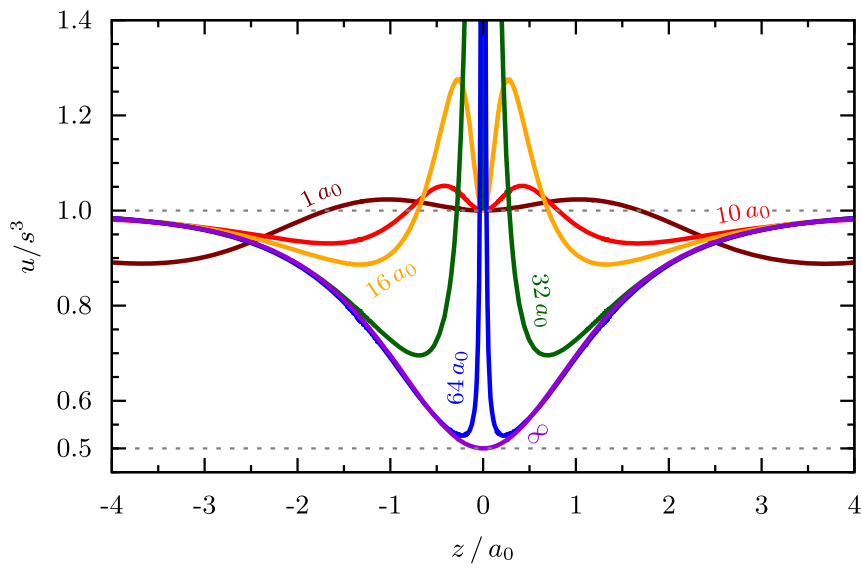

FIG. 5. Cross section of $u / s^{3}$ evaluated in the minimal model of a nodal plane. 


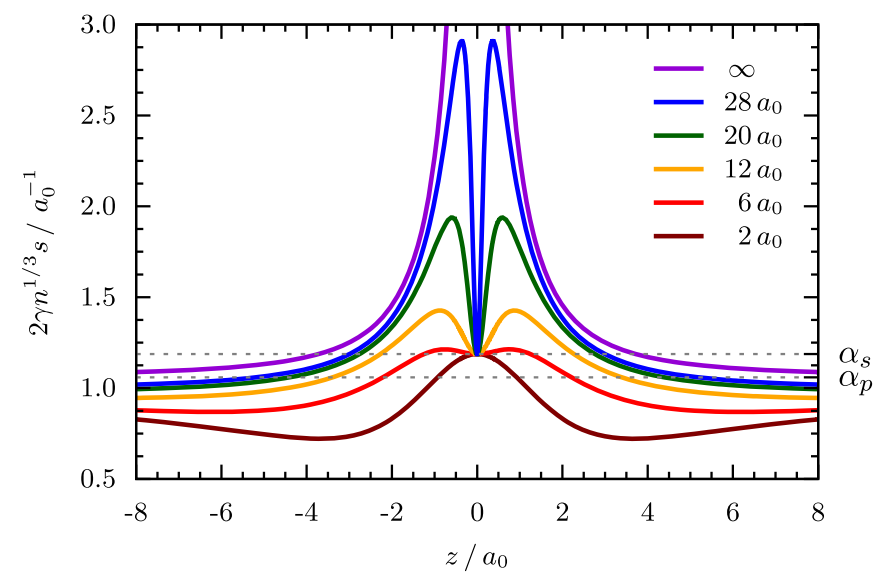

FIG. 6. Cross section of $2 \gamma n^{1 / 3} s$ evaluated in the minimal model of a nodal plane.

demonstrates. Far from the nodal plane, i.e., for $|z| \rightarrow \infty$, $2 \gamma n^{1 / 3} s$ approaches the system-dependent constant $\alpha_{p}$, which is given by the homo as expected by Eq. (7). Once more, exactly on the nodal plane the quantity is solely determined by the $s$ contribution and therefore approaches $\alpha_{s}$, the analogous constant of the underlying $s$ orbital. As the neighborhood affected by this limit contracts to the nodal plane as $r \rightarrow \infty$, the semilocal ratio approaches its behavior on the pure nodal plane, which is given by

$$
2 \gamma n^{1 / 3} s \sim \frac{2}{z}+\left(\frac{1}{4}-\frac{1}{\alpha_{p} r}\right) \alpha_{p}^{2} z^{2}
$$

as $z \rightarrow 0^{+}$. Hence, unlike $t / s^{2}$ or $u / s^{3}, 2 \gamma n^{1 / 3} s$ is not almost everywhere smooth as $r \rightarrow \infty$, but it develops a pole of first order: its denominator $n$ has a double root on the pure nodal plane, while its numerator $|\nabla n|$ exhibits only a single root. Therefore, even infinitely far outside of the molecule the region affected by the nodal plane maintains a finite width for this quantity.

Finally, the semilocal quantity $2 \sqrt{2 \tau / n}$, which is key to the BJ potential, is examined. It is shown in Fig. 7. The kinetic energy density, $\tau$, is constructed in analogy to the density of

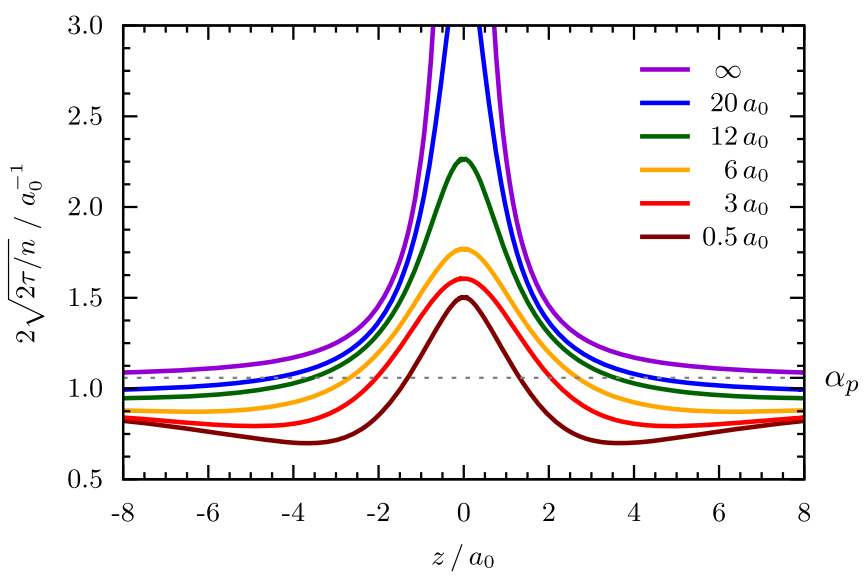

FIG. 7. Cross section of $2 \sqrt{2 \tau / n}$ evaluated in the minimal model of a nodal plane.
Eq. (1) by evaluating Eq. (4) with the orbitals of the minimal model. Since the prefactor is chosen such that the quantity approaches the same spherical symmetric limit as $2 \gamma n^{1 / 3} s$, the behavior of $2 \sqrt{2 \tau / n}$ is similar to this quantity for $|z| \rightarrow \infty$ and the system-dependent constant $\alpha_{p}$ is likewise approached far outside the nodal surface. Exactly on the nodal plane, however, $2 \sqrt{2 \tau / n}$ does not approach the constant $\alpha_{s}$, but it diverges exponentially along the plane, as the $p$ contribution to $\tau$ does not vanish. Because the pure model, which is approached as $r \rightarrow \infty$ almost everywhere, is determined by a single orbital only, $2 \sqrt{2 \tau / n}$ equals $2 \gamma n^{1 / 3} s$ strictly in this limit, as $\tau^{W}=$ $|\nabla n|^{2} / 8 n$ is the single orbital limit of $\tau$. Hence, on the pure nodal plane

$$
2 \sqrt{\frac{2 \tau}{n}} \sim \frac{2}{z}+\left(\frac{1}{4}-\frac{1}{\alpha_{p} r}\right) \alpha_{p}^{2} z^{2}
$$

as $z \rightarrow 0^{+}$, which features a pole of first order, implying once more a region of finite width affected by the nodal plane as $r \rightarrow$ $\infty$. Further insight into the consequences that nodal surfaces have when $\tau$ is used as a part of an iso-orbital indicator, e.g., in the context of local hybrid functionals [59], is discussed in Ref. [49].

\section{RESULTS FOR THE ASYMPTOTICS OF SEMILOCAL POTENTIALS}

We now evaluate the behavior of semilocal potentials in the vicinity of a nodal surface based on the minimal model. We focus on two aspects: First, we examine the behavior exactly along the nodal plane, which is described by the complete minimal model. While this gives insight into whether and how rapidly the potential diverges along the nodal plane, the affected region might be arbitrarily small and might contract with increasing distance from the center of the system $r$. Therefore, secondly, the pure model is used to classify the impact of the nodal surface on its neighborhood as $r \rightarrow \infty$.

\section{A. The BJ potential functional in the vicinity of nodal surfaces}

Various modifications of the BJ exchange potential functional are used quite frequently in the literature [29-35]. Therefore, we begin our application of the minimal model with the BJ expression. The BJ functional directly models the exchange potential as a sum of the Slater exchange potential [60] and a term expressed in the kinetic energy density,

$$
v_{\mathrm{x}}^{\mathrm{BJ}}=v_{\mathrm{x}}^{\text {Slater }}+C_{\Delta v} \sqrt{\frac{2 \tau}{n}},
$$

where $C_{\Delta v}=\sqrt{5 / 12} / \pi$. As it is a potential functional, it has the major drawback that no corresponding exchange functional exists [24,61-63]. For a regular decaying density in the sense of Eq. (5), the correction term to the Slater potential approaches its characteristic positive asymptotic constant,

$$
v_{\mathrm{x}}^{\mathrm{BJ}} \sim \frac{C_{\Delta v}}{2} \alpha,
$$

as $|\mathbf{r}| \rightarrow \infty$

Since the Slater potential vanishes $\propto-1 /|\mathbf{r}|$ isotropically even in the presence of nodal surfaces, we can restrict the investigation to the correction term, which is proportional 
to the semilocal quantity $2 \sqrt{2 \tau / n}$ of the last paragraph. Therefore, we can conclude that the BJ potential diverges exponentially along the nodal plane; insertion of the model density yields

$$
v_{\mathrm{x}}^{\mathrm{BJ}}(r, z=0) \sim C_{\Delta v} \sqrt{\frac{n_{p}^{0}}{n_{s}^{0}}} \exp \left[\left(\alpha_{s}-\alpha_{p}\right) r / 2\right] .
$$

Hence, the rate of the exponential divergence is given by the difference of the decay parameters of the homo, $\alpha_{p}$, and that of the underlying orbital, $\alpha_{s}$, which in turn are closely connected to the corresponding eigenvalues. A combination of Eq. (11) with the asymptotics of the Slater potential gives the behavior of the BJ potential in the vicinity of the pure nodal plane,

$$
v_{\mathrm{x}}^{\mathrm{BJ}} \sim \frac{C_{\Delta v}}{z}-\frac{1}{r}+O\left(z^{2}\right)
$$

as $z \rightarrow 0^{+}$. Therefore, the divergence of the BJ potential affects a finite region enclosing the nodal plane and does not contract to a set of zero measure as $r \rightarrow \infty$. A visualization of the asymptotic BJ potential in the vicinity of a nodal plane was essentially given in Fig. 7.

While it has already been noted in the original work that the BJ potentials diverges at orbital nodes, and should consequently be used with ground-state configurations only [28], our analysis aggravates these observations, as it shows them to be relevant for ground-state configurations as well.

\section{B. AK13 in the vicinity of nodal surfaces}

We now turn to the AK13 functional, which is based on the usual GGA form of the exchange energy,

$$
E_{\mathrm{x}}^{\mathrm{GGA}}=A_{\mathrm{x}} \int n^{4 / 3} F(s) d^{3} r .
$$

In the case of AK13, the enhancement factor $F(s)$ is given by

$$
\begin{aligned}
F^{\mathrm{AK} 13}(s)=1+B_{1} s & \ln (1+s) \\
& +B_{2} s \ln [1+\ln (1+s)],
\end{aligned}
$$

where the constants $B_{1}=2 / 27+8 \pi / 15$ and $B_{2}=4 / 81-$ $8 \pi / 15$ have been determined in a nonempirical fashion. Its key feature is that its corresponding potential, i.e., the functional derivative of Eq. (16),

$$
\begin{aligned}
v_{\mathrm{x}}^{\mathrm{GGA}}= & A_{\mathrm{x}} \frac{4}{3} n^{1 / 3}\left[F(s)-s \partial_{s} F(s)+\frac{3}{4}\left(\frac{u}{s^{3}}-\frac{t}{s^{2}}\right) s \partial_{s} F(s)\right. \\
& \left.+\left(1-\frac{3}{4} \frac{u}{s^{3}}\right) s^{2} \partial_{s}^{2} F(s)\right]
\end{aligned}
$$

typically approaches a positive system-dependent constant outside of a finite system. This novel GGA feature was inspired by the BJ exchange potential that we investigated in Sec. IV A. The asymptotic constant of the AK13 potential,

$$
v_{\mathrm{x}}^{\mathrm{AK} 13} \sim-\frac{A_{\mathrm{x}} B_{1}}{6 \gamma} \alpha
$$

as $|\mathbf{r}| \rightarrow \infty$, follows formally from inserting the asymptotic relations for a regular decaying density, Eq. (7), together with the asymptotic enhancement factor of AK13 as $s \rightarrow \infty$ in leading order,

$$
F^{\mathrm{AK} 13}(s) \sim B_{1} s \ln (s)+B_{2} s \ln [\ln (s)] .
$$

Hence, the asymptotic constant is related to the precise leading term in the divergent enhancement factor $F(s) \propto s \ln (s)$ as $s \rightarrow \infty$, which we will refer to as "critical asymptotic." It is the threshold between asymptotic GGA potentials that are, for a regularly decaying density, vanishing and diverging in the limit $|\mathbf{r}| \rightarrow \infty$.

To discuss the behavior of a GGA potential in the vicinity of a nodal plane, Eq. (18) has to be examined in the limit $s \rightarrow \infty$ as well. The key difference is that one has to consider the altered relations of $n, s, t$, and $u$ due to the presence of the nodal surface, which we have discussed in Sec. III. In particular, it was shown that the Laplacian contribution to the GGA potential is dominant exactly along the nodal plane. Therefore, the asymptotic of the GGA potential along the plane is given by

$$
v_{\mathrm{x}}^{\mathrm{GGA}}(r, z=0) \sim-A_{\mathrm{x}} n^{1 / 3} \frac{t}{s} \partial_{s} F(s) .
$$

Inserting the asymptotic enhancement factor of AK13 as $s \rightarrow \infty$ in leading order given by Eq. (20), i.e., $\partial_{s} F^{\mathrm{AK} 13}(s) \sim$ $B_{1} \ln (s)$, and using the density of the minimal model, we find

$$
v_{\mathrm{x}}^{\mathrm{AK} 13}(r, z=0) \sim-\frac{A_{\mathrm{x}} B_{1} r}{3 \gamma}\left(\frac{n_{p}^{0}}{n_{s}^{0}}\right) \exp \left[\left(\alpha_{s}-\alpha_{p}\right) r\right] .
$$

Thus, the AK13 potential diverges exponentially along nodal surfaces at twice the rate of the BJ potential; cf. Eq. (14).

To evaluate the behavior of the AK13 potential in the neighborhood of the nodal plane as $r \rightarrow \infty$, we use the pure minimal model: Inserting relations (8)-(10) into the asymptotic GGA potential, deduced from Eq. (18), yields

$$
v_{\mathrm{x}}^{\mathrm{AK}} \sim-\frac{A_{\mathrm{x}} B_{1}}{2} n^{1 / 3} s \sim-\frac{A_{\mathrm{x}} B_{1}}{2 \gamma} \frac{1}{z}
$$

as $z \rightarrow 0^{+}$. Hence, far from the center of the molecule, the AK13 potential behaves similar to the BJ potential, i.e., as if it had a pole of first order on the nodal plane; even the amplitude of the pole is approximately of the same strength. Consequently, the region affected by the nodal plane has a fixed width and does not contract to a set of zero measure in the case of the AK13 potential, either. Figure 8 visualizes the AK13 potential in the vicinity of the nodal plane via cross sections.

In addition to the features that we just discussed, Fig. 8 demonstrates that local minima surrounding the nodal surface show up in the AK13 potential. To explain the mechanism behind them, we have to revisit the asymptotic GGA potential and note that the AK13 construction relies on the cancellation of the first-order terms, i.e., contribution to the potential $\propto$ $s \ln (s)$. To achieve the cancellation, the asymptotic limits of $t / s^{2}$ and $u / s^{3}$ have to be equal, as they are in the spherical symmetric case and exactly on a pure nodal surface. However, for a fixed distance to the nodal surface $z>0$ the limits of these semilocal ratios differ slightly as $r \rightarrow \infty$, as a comparison of the second-order terms in the asymptotic relations (8) and (9) readily demonstrates. Consequently, the leading-order terms 


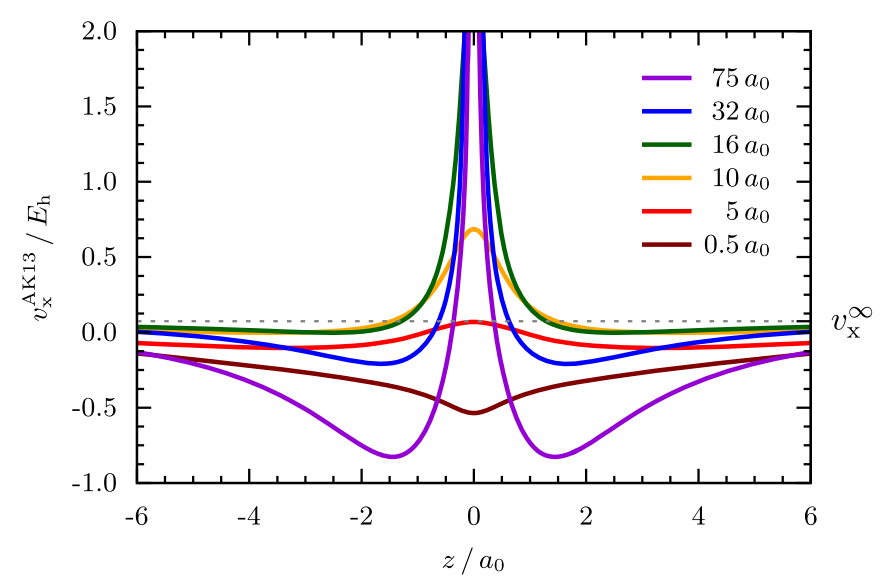

FIG. 8. Cross sections of the AK13 potential at different $r$ values evaluated in the neighborhood of the nodal surface in the minimal model. Exactly at the nodal plane $(z=0)$ the potential diverges exponentially as $r \rightarrow \infty$, being surrounded by an area where the potential behaves $\propto 1 / z$. Given a finite $r$ value, the potential approaches the constant $v_{x}^{\infty}$ for $z \rightarrow \infty$. Additionally, with an increasing $r$ value, local minima surrounding the nodal surface show up, as the potential diverges linearly in $r$ toward negative infinity for every finite $z \neq 0$.

remain, i.e.,

$$
v_{\mathrm{x}}^{\mathrm{AK} 13} \sim\left[\lim _{r \rightarrow \infty}\left(\frac{u}{s^{3}}-\frac{t}{s^{2}}\right)\right] \frac{A_{\mathrm{x}} B_{1}}{6 \gamma} \alpha_{p}^{2} r
$$

as $r \rightarrow \infty$ for $z>0$ fixed. This implies a linearly diverging potential. Hence, except for right on the nodal surface (a set of zero measure where the potential diverges exponentially to positive infinity), the AK13 potential approaches negative infinity linearly in $r$ with a $z$-dependent slope. This results in a further amplification of the divergence. A nodal behavior very similar to the AK13 potential is to be expected of the potentials of exchange-enhanced GGAs [64] due to their usage of the same $s \ln (s)$ asymptotics.

\section{B88 in the vicinity of nodal surfaces}

Next, we turn to GGAs with enhancement factors $F(s) \ll$ $s \ln (s)$ as $s \rightarrow \infty$, i.e., identified above as subcritical asymptotics. The potential of these GGAs will vanish with increasing distance from a finite spherically symmetric system. However, we will demonstrate that this condition is not sufficient to avoid the divergence of the corresponding potential along nodal surfaces. We discuss this issue with the widely used B88-GGA serving as an example. To reproduce the correct asymptotic behavior of the exact exchange-energy density, the B88 enhancement factor diverges slightly slower than that of AK13,

$$
F^{\mathrm{B} 88}(s) \sim-\frac{\gamma}{3 A_{\mathrm{x}}} \frac{s}{\ln (s)}
$$

as $s \rightarrow \infty$, causing the corresponding potential to vanish $\propto$ $-1 /|\mathbf{r}|^{2}[65]$ in the asymptotic region outside of nodal surfaces. Utilizing Eq. (21) and the minimal model gives the asymptotic

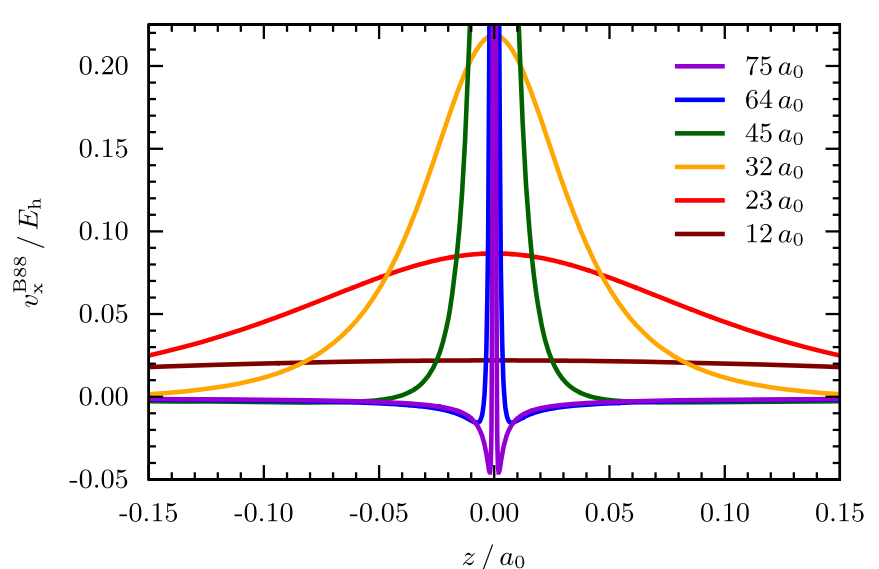

FIG. 9. Cross sections of the B88 potential at different $r$ values evaluated in the immediate vicinity of the nodal surface in the minimal model. Exactly at the nodal plane $(z=0)$ the potential diverges exponentially as $r \rightarrow \infty$, being surrounded by an area where the potential behaves $\propto-1 /\left[z \ln ^{2}(z)\right]$ (visible from $64 a_{0}$ onward), which in turn contracts $\propto 1 / r^{2}$ as $r \rightarrow \infty$.

behavior of the B88 potential along the nodal plane,

$$
v_{\mathrm{x}}^{\mathrm{B} 88}(r, z=0) \sim \frac{1}{\alpha_{s}^{2} r}\left(\frac{n_{p}^{0}}{n_{s}^{0}}\right) \exp \left[\left(\alpha_{s}-\alpha_{p}\right) r\right]
$$

as $r \rightarrow \infty$. Consequently, the B88 potential diverges slightly slower than the AK13 potential along nodal surfaces, while maintaining the same exponential rate, which is twice the rate of the BJ potential.

On the pure model and close to the nodal plane, the leadingorder terms cancel and we find

$$
v_{\mathrm{x}}^{\mathrm{B} 88} \sim-\frac{\gamma}{6} \frac{n^{1 / 3} s}{\ln ^{2}(s)}
$$

as $s \rightarrow \infty$ and for $|z| \ll 1$. While the numerator $n^{1 / 3} s$ features a pole of first order in $z$ [cf. Eq. (10)], the denominator is ambivalent, as, on the one hand,

$$
\ln (s) \sim-\frac{5}{3} \ln (z)
$$

as $z \rightarrow 0^{+}$for fixed $r$, and, on the other hand,

$$
\ln (s) \sim \alpha_{p} r / 3
$$

as $r \rightarrow \infty$ for fixed $z>0$. Therefore, the B88 exchange potential exhibits a negative pole on the pure nodal surface,

$$
v_{\mathrm{x}}^{\mathrm{B} 88} \sim-\frac{3}{50} \frac{1}{z \ln ^{2}(z)}
$$

as $z \rightarrow 0^{+}$, but the region affected by the pole contracts, as for fixed $z>0$ the quantity $n^{1 / 3} s$ approaches a constant as $r \rightarrow \infty$; cf. Fig. 6. Therefore, the potential vanishes,

$$
v_{\mathrm{x}}^{\mathrm{B} 88} \sim-\frac{3}{2} \gamma n^{1 / 3} s \frac{1}{\alpha_{p}^{2} r^{2}}
$$

as $r \rightarrow \infty$ for a fixed $z>0$. All of these aspects are visualized in Fig. 9 by cross sections of the B 88 potential in the immediate vicinity of the nodal plane. 
The exchange parts of the AM05 functional [66] as well as QrevLB94 [67] are expected to be similar to B88 with respect to their nodal surface properties, because they share the asymptotic enhancement factor $\propto s / \ln (s)$ with B88.

\section{LB94 potential functional in the vicinity of nodal surfaces}

The final potential we wish to discuss in this context is the exchange model potential of van Leeuwen and Baerends (LB94) [17], as it also exhibits irregularities, but ones that are qualitative different from the ones of AK13, BJ, or B88. The LB94 model potential is designed as a semilocal correction to the exchange LDA potential based on $n$ and $s$, with the aim to incorporate the correct $-1 /|\mathbf{r}|$ asymptotic as well as an atomic-shell structure. In the asymptotic region, i.e., for $s \rightarrow \infty$, the LB94 model potential is generally described by the relation

$$
v_{\mathrm{x}}^{\mathrm{LB} 94} \sim-\frac{2 \gamma}{3} \frac{n^{1 / 3} s}{\ln (s)}
$$

even along or in the vicinity of nodal surfaces. Importantly, due to its model potential character, the LB94 potential does not depend on the Laplacian of the density, in contrast to GGA potentials that are functional derivatives. Consequently, the LB94 potential does not diverge exponentially exactly along a nodal plane, but vanishes there in Coulombic fashion $\propto-1 / r$, and likewise in any other direction. Nevertheless, the LB94 potential features divergent behavior in a region enclosing the nodal plane. This can best be understood by using the pure minimal model, which gives the asymptotic relation

$$
v_{\mathrm{x}}^{\mathrm{LB} 94} \sim \frac{2}{5} \frac{1}{z \ln (z)}
$$

as $z \rightarrow 0^{+}$, and it describes a negative pole of the LB94 potential on the pure nodal plane. In the full model, these poles translate into minima of the LB94 potential surrounding the nodal plane at intermediate distances. As $r \rightarrow \infty$, the depth of these minima grows without bounds as the position of the minima converges exponentially to the nodal plane. Yet, in the same limit the region affected by the minima contracts as the LB94 potential vanishes,

$$
v_{\mathrm{x}}^{\mathrm{LB} 94} \sim-2 \gamma n^{1 / 3} s \frac{1}{\alpha_{p} r}
$$

for a fixed $z>0$. This follows from the same arguments as in the discussion of the B88 potential, i.e., for fixed $z>0$ the quantity $n^{1 / 3} s$ approaches a constant while $\ln (s)$ shows a linear behavior in $r$. Figure 10 visualizes these findings for the LB94 potential, once more via cross sections in the vicinity of the nodal plane. We note that the nodal surface behavior of the model potentials of Lembarki et al. [68] can be expected to be similar to that of LB94.

\section{E. Potential landscapes of semilocal potentials}

For a final comparison of the behavior in response to nodal surfaces of all exchange potentials that were discussed in this article, we have plotted these potentials along the nodal plane of the minimal model in Fig. 11 and the landscapes of the potentials in Fig. 12. Figure 11 confirms the asymptotic relations of Eqs. (14), (22), and (26), while the EXX potential

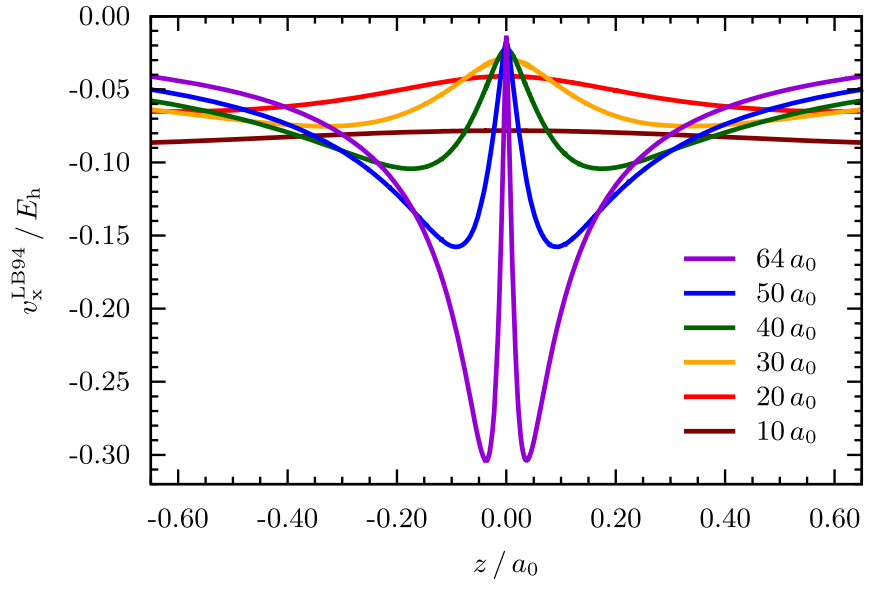

FIG. 10. Cross sections of the LB94 potential at different $r$ values evaluated in the vicinity of the nodal surface in the minimal model. Exactly at the nodal plane $(z=0)$, the potential does not diverge but vanishes $\alpha-1 / r$, being surrounded by a divergent area where the potential behaves $\propto 1 /[z \ln (z)]$, which in turn contracts $\propto 1 / r$ as $r \rightarrow \infty$.

tends to a finite positive value in the same limit. Whereas the B88 potential diverges formally faster than the BJ potential, this is only relevant in the far asymptotic region, which is typically not part of numerical calculations.

Figure 12 displays the effect of the nodal plane on all four exchange potentials within the typical spatial range of a numerical calculation, and it should be compared to the EXX potential in Fig. 3. Aside from the (irregular) behavior exactly along the nodal plane, the landscapes demonstrate how the width of the affected region differs. In the case of EXX, the region affected by the nodal plane, i.e., the ridge, contracts exponentially with increasing distance from the center of the system. The closest to this ideal is the B88 potential, though the affected region contracts even faster. Therefore, the

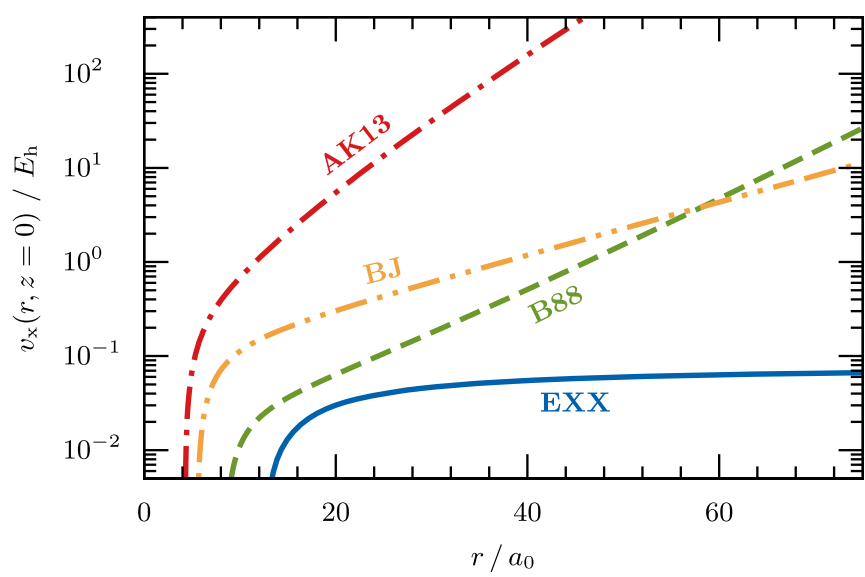

FIG. 11. Exchange potentials along the nodal surface of the homo $(z=0)$ in the minimal model. While the EXX potential [in the Krieger-Li-Iafrate (KLI) approximation [56]] reaches a positive constant, the AK13, B88, and the BJ potentials diverge exponentially as $r \rightarrow \infty$. The LB94 potential is not visible in this figure, as it behaves regularly exactly on the nodal plane and approaches zero from below. 


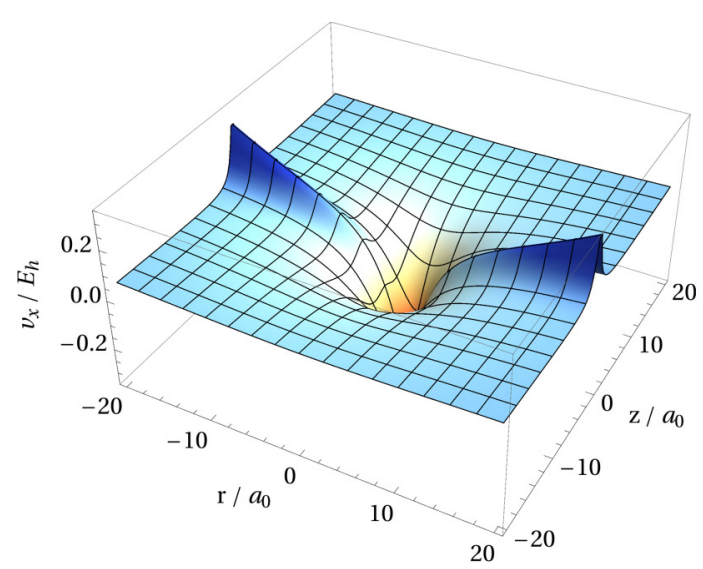

(a) B.J

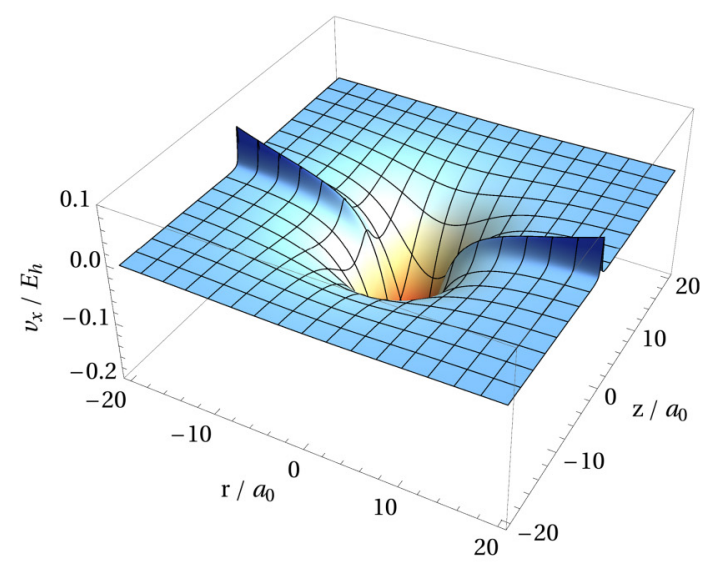

(c) B88

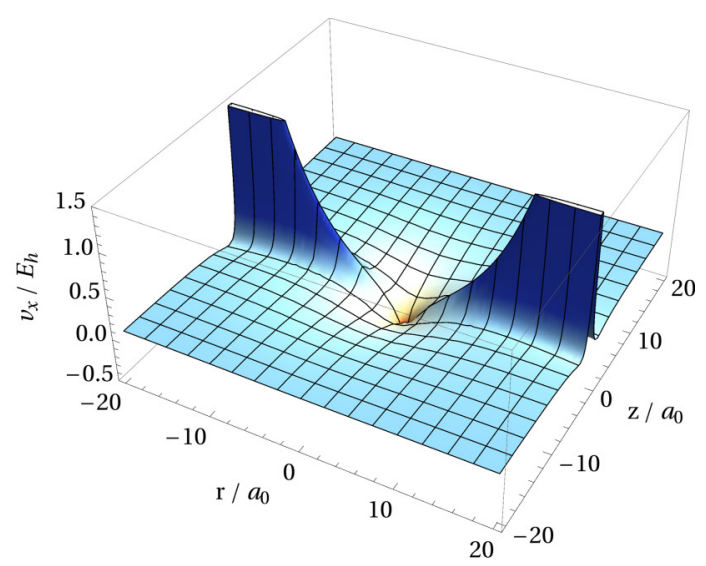

(b) AK13

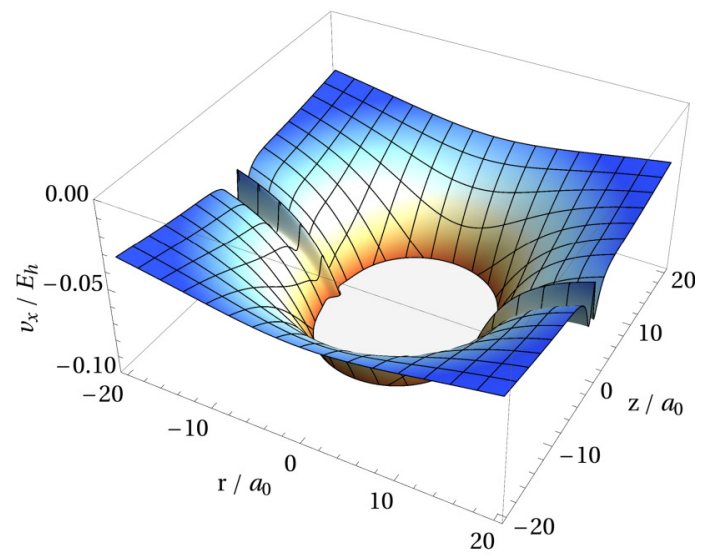

(d) LB94

FIG. 12. Semilocal exchange potentials in the minimal model of a nodal plane located at $z=0$. Note that the scale of the potential axis as well as the coloring visualizing the height of the potentials are not standardized. For a comparison to EXX, see Fig. 3. Except for LB94, all exchange potentials diverge exponentially along the nodal plane, though with different magnitude and width. All potentials were evaluated and plotted using MATHEMATICA [57].

numerical resolution of the B88 potential irregularity is highly questionable in practice, unless grid points exactly along the nodal plane are used. In the case of BJ, the region affected by the nodal plane is qualitatively different, as it does not contract but approaches a finite width as $r \rightarrow \infty$. The AK13 potential is similar in principle, though one could argue that in the case of AK13 the width is even expanding as minima surrounding the divergent region grow without limits. It is noteworthy that even though the GGA potentials of AK13 and B88 diverge along the nodal plane, their corresponding exchange energies per volume remain finite throughout.

\section{FORMAL CONSTRAINTS FOR WELL-BEHAVED GGA POTENTIALS}

The rate of divergence of a GGA potential for exchange along the nodal plane is determined by the leading power $d$ of the asymptotic enhancement factor, $F(s) \propto s^{d}$ as $s \rightarrow \infty$ neglecting logarithmic contributions, i.e., $F(s) \sim C s^{d} \ln ^{c}(s) \propto$ $s^{d}$ regardless of the precise value of $C$ and $c$; consequently, in the case of AK13 and B88, $d=1$. For more general enhancement factors, $d$ may be defined via

$$
d=\inf \left\{b \in \mathbb{R} \mid \lim _{s \rightarrow \infty} F(s) / s^{b}=0\right\} .
$$

Additionally, if $F(s)$ approaches a finite constant $F(\infty)$ as $s \rightarrow \infty$, one should replace $F(s)$ in the equations above by $[F(s)-F(\infty)]$, which then corresponds to a negative leading power $d<0$.

One can show that in general

$$
v_{\mathrm{x}}^{\mathrm{GGA}}(r, z=0) \propto \exp \left[\left(\frac{2+d}{3} \alpha_{s}-\alpha_{p}\right) r\right]
$$

based on Eq. (21) and the insight that $\nabla^{2} n$ is in contrast to $n$ and $\nabla n$ governed by the $p$ orbital exactly along the nodal plane as $r \rightarrow \infty-$ a detailed derivation can be found in Appendix A. Thus, avoiding a divergence along a nodal surface requires

$$
d \leqslant-2+3 \frac{\alpha_{p}}{\alpha_{s}}=: d_{c},
$$

or system-independently and therefore strictly formulated,

$$
d \leqslant-2 \text {. }
$$

Consequently, $F(s) \sim C_{1}+C_{2} / s^{2}$ as $s \rightarrow \infty$ is a sufficient condition to avoid a divergence along a nodal surface, while an asymptotic enhancement factor in the range $-2<d<1$ will diverge in some systems with a nodal surface, but not in all. Note that for exactly $d=0$ without any logarithmic 
contribution there is no divergence, because the proportionality constant in Eq. (36) vanishes trivially. The system-specific threshold value $d_{c}$ depends on the difference between the highest and second highest occupied orbital eigenvalues. While a nearly degenerate situation corresponds to $d_{c} \approx 1$, the lower limit $d_{c}=-2$ is approached in the case of a weakly bound highest occupied orbital on top of a strongly bound second highest occupied orbital. The threshold of our minimal model is, for instance, $d_{c} \approx 0.775$ and thus describes a rather degenerate case.

Exchange GGAs that we therefore expect to show a systemdependent exponential divergence of the potential along the nodal planes are, e.g., PW86 [69] and B86b [70] with $d=2 / 5$ as well as the Local Airy gas approximation (LAG) [71] with $d \approx 0.9$. Additionally, GGAs for exchange that are designed to satisfy the correct nonuniform coordinate scaling limit [72,73] via $F(s) \sim C s^{-1 / 2}$ as suggested in Ref. [74] might in rare cases $\left(\alpha_{s}>2 \alpha_{p}\right)$ lead to an exponential divergence of the corresponding potential along the nodal plane as well.

In addition to the exponential divergence along the nodal plane, irregular behavior in the neighborhood of the nodal plane has to be considered. To avoid this, i.e., a pole on the pure model of the nodal plane, the leading asymptotic power of the enhancement factor $d, F(s) \propto s^{d}$ as $s \rightarrow \infty$ neglecting logarithmic contributions, has to be in general less than or equal to $2 / 5$. This follows from Eq. (18), when using the asymptotic relations Eqs. (8) and (9) via

$$
v_{\mathrm{x}}^{\mathrm{GGA}} \propto n^{1 / 3} s^{d} \propto z^{(2-5 d) / 3}
$$

as $z \rightarrow 0^{+}$. As this requirement is naturally included in the constraint of Eq. (38), i.e., $d \leqslant-2$, the latter is sufficient to avoid any irregular behavior of a GGA potential in the vicinity of nodal surfaces. It follows from this argument that several widely used semilocal functionals for exchange are free of any irregular behavior in the vicinity of nodal surfaces, as they fulfill this sufficient condition. Among these are LDA, B86a [75], and PBE [76].

A rather far-reaching consequence of this analysis is that for the GGA exchange form, the design criteria of either a nonvanishing asymptotic constant in the potential or the correct asymptotic Coulombic behavior of the potential or of the energy density are all incompatible with the regular behavior of the potential in the vicinity of asymptotic nodal surfaces. The detailed line of arguments that leads to this conclusion is given in Appendix B.

\section{DISCUSSION}

While many of the results of the previous sections were derived utilizing a model system, we expect them to be at least qualitatively transferable to true nodal surfaces of real molecules or atoms. Our reasoning is that the behavior of the density in the vicinity of an asymptotic nodal surface is universal in the sense that it essentially consists of two additive contributions. Both contributions vanish exponentially, but with different decay lengths. Whereas the slower decaying contribution is smooth and nodeless in the asymptotic region, the faster decaying contribution features a nodal surface, which is likely to be approximate harmonically in terms of the distance to the nodal surfaces. Since these conditions are sufficient to derive all presented results to leading order and are satisfied by our minimal model, our results are of general relevance. For example, our arguments also apply to other nodal surfaces as, e.g., generated by higher spherical harmonics. In the latter case, all results will be maintained qualitatively, as well as quantitatively in leading order, when one measures the distance to a specific nodal surface by $z$, and the coordinate along the nodal surface by $r$. One may also specifically wonder how our arguments change if one replaces the $p_{z}$ orbital in our model by the linear combination $p_{x}+i p_{y}$, changing the homo density into a torus. In this case, the nodal plane would reduce to a single line, the $z$ axis. Yet, except for an interchange of $r$ and $z$, the results and plots would look nearly indistinguishable to the ones that we present here. In addition to these arguments, the relevance of our findings is also evident from the divergences that are observed in real systems, as demonstrated, e.g., in Fig. 1 for the benzene molecule and the AK13 functional.

We observe that all functionals that we know of that incorporate in a semilocal fashion either a system-dependent asymptotic constant or the correct asymptotic Coulombic behavior of the potential or the energy density are affected by nodal surfaces issues-this list even includes Laplacian based meta-GGA constructions [19,77]. Additionally, we attempted to combine any of these asymptotic criteria with regular behavior on nodal surfaces in density functionals of rather general semilocal form-but without success so far. For the GGA exchange form in particular, such asymptotics are incompatible with a potential that behaves regularly in the vicinity of asymptotic nodal surfaces; cf. Appendix B. This is in line with the observation that commonly used semilocal functionals do not perform well when the exact xc hole is not localized around its electron. The hole-localization condition is radically violated in the asymptotic limit, where the electron is far out but its hole remains well inside the system. Therefore, the above-mentioned asymptotic features are very challenging design criteria for semilocal functionals.

The exponentially diverging semilocal exchange potentials of AK13, BJ, B88, and LB94 have several potentially unpleasant implications. Diverging potentials are suspicious from a conceptual perspective. Even though it was found that the EXX potential approaches a positive constant [38-41] in the direction of nodal surfaces, its implications and the behavior of the combined xc potential are still controversially discussed $[47,48]$. Adopting a positive perspective, one may interpret the divergences that we discuss here as a contribution to this discussion.

However, in terms of the practical application of these semilocal functionals, their irregular behavior is problematic. In particular, when the irregularities are confined to very narrow regions of space, such as, e.g., in the case of B88, a numerical representation in terms of some chosen basis set may not resolve the divergence. In this case, the numerical calculation may converge without problems. Strictly speaking, however, such a calculation has concealed a feature that is part of the proper functional derivative. On the other hand, choosing the numerical resolution such that it captures the divergence can have detrimental consequences: As a divergence of these potentials is generated in a semilocal fashion, small, normally insignificant changes of the density in the asymptotic region 
close to a nodal surface can cause tremendous feedback on these potentials. Hence, in an iterative KS calculation, a positive feedback loop for irregularities and numerical instabilities can arise, impeding a self-consistent solution of the KS equations. We find this to be very much the case for our own attempts at converging AK13 results for systems with nodal surfaces, and also other authors have reported numerical problems with self-consistent calculations [78]. Furthermore, unusual oscillations in the AK13 potential have also been observed in the interstitial region of Si crystals [35], and these might be another consequence of the features that we discussed here for finite systems. Quite generally, one might speculate that similar issues could have influenced the convergence of some of the many published values obtained with B88- and $\mathrm{BJ}$-based potential functionals.

\section{SUMMARY AND CONCLUSIONS}

We have introduced and used a minimal model of general utility to examine exchange potentials along nodal surfaces of the highest occupied orbital. The model was used to investigate the corresponding potentials from the exchangeenergy functionals AK13 and B88, as well as the BJ and LB94 model exchange potentials. We commented on several other functionals that are expected to have nodal surface properties in close similarity to these four paradigm cases. None of these potentials is well-behaved in the vicinity of a nodal surface, but rather they diverge exponentially. The AK13 functional has the most strongly divergent potential, which appears to prevent numerical convergence in practical calculations on molecular systems with nodal surfaces. The present work gives results and tools that should be useful for the investigation of other functional constructs, and for creating future expressions that avoid nodal surface anomalies. In particular, we derived a condition that is sufficient for avoiding nodal surface problems, and we pointed out that, e.g., LDA, B86a, and PBE fulfill this condition. Our results are relevant for the developers of density functionals, and also for users of DFT: Divergent potentials pose a challenge for the numerical convergence of solutions to the KS equations. Our work serves to caution that great care has to be taken in calculations with functionals that show special features close to nodal surfaces.

\section{ACKNOWLEDGMENTS}

S.K. and T.A. acknowledge financial support by the German-Israeli Foundation for Scientific Research and Development. T.A. acknowledges support by the University of Bayreuth Graduate School. R.A. acknowledges financial support from the Swedish Research Council (VR), Grant No. 2016-04810 and the Swedish e-Science Research Centre (SeRC). S.K. acknowledges a discussion with W. Kohn about the question of an "exact exchange perpetuum mobile."

\section{APPENDIX A: ASYMPTOTICS OF GGA POTENTIALS EXACTLY ALONG THE NODAL PLANE OF THE MINIMAL MODEL}

Here we provide a detailed derivation of Eq. (36), i.e., rate of divergence for GGA potentials exactly along a nodal plane of the minimal model: As we have demonstrated in Sec. III, the Laplacian of the density is the only ingredient of the GGA potential of Eq. (18) whose $p$ contribution does not vanish exactly on the nodal plane and therefore dominates,

$$
\left(\nabla^{2} n\right)(r, z=0) \sim\left(\nabla^{2} n_{p}\right)(r, z=0) \propto \exp \left(-\alpha_{p} r\right)
$$

in the limit $r \rightarrow \infty$. On the contrary, the $p$ contribution to $n$, $\nabla n$, and $\nabla|\nabla n|$ vanishes at $z=0$, thus their dominant behavior as $r \rightarrow \infty$ is determined by the exponential decay of the $s$ orbital,

$$
\begin{aligned}
n(r, z=0) & \propto(\nabla n)(r, z=0) \propto(\nabla|\nabla n|)(r, z=0) \\
& \propto n_{s}(r, z=0) \propto \exp \left(-\alpha_{s} r\right) .
\end{aligned}
$$

Because the exponential divergence of GGA potentials along nodal planes stems from the occurrence of these different decay lengths, it is given by Eq. (21),

$$
v_{\mathrm{x}}^{\mathrm{GGA}}(r, z=0) \sim-A_{\mathrm{x}} n^{1 / 3} \frac{t}{s^{2}} s \partial_{s} F(s),
$$

where we have to consider $F(s)$ in the limit $s \rightarrow \infty$, as this limit is approached for $r \rightarrow \infty$ on the nodal plane (just as in the spherically symmetric case for $|\mathbf{r}| \rightarrow \infty)$. Now, we consider a general asymptotic enhancement factor in this limit,

$$
F(s) \sim C s^{d} \ln ^{c}(s),
$$

where $C, d$, and $c$ are arbitrary constants. Therefore,

$$
v_{\mathrm{x}}^{\mathrm{GGA}}(r, z=0) \sim-A_{\mathrm{x}} C d n^{1 / 3} \frac{t}{s^{2}} s^{d} \ln ^{c}(s) .
$$

Concerning the exponential divergence, we can neglect the prefactor and the logarithm as $\ln ^{c}(s)$ is only polynomial in $r$. Inserting the definitions of $s$ and $t$ [cf. Eq. (3)] and using relations (A1) and (A2) gives the final result,

$$
\begin{aligned}
v_{\mathrm{x}}^{\mathrm{GGA}}(r, z=0) & \propto n^{1 / 3} t s^{d-2} \\
& \propto n^{1 / 3-5 / 3-(d-2) 4 / 3}\left(\nabla^{2} n\right)|\nabla n|^{d-2} \\
& \propto n_{s}^{-(d+2) / 3} n_{p} \propto \exp \left[\left(\frac{2+d}{3} \alpha_{s}-\alpha_{p}\right) r\right],
\end{aligned}
$$

which was presented in Eq. (36) and discussed thereupon.

\section{APPENDIX B: INCOMPATIBILITY OF ASYMPTOTIC SEMILOCAL DESIGN CRITERIA WITH NODAL SURFACES}

Under the assumption of a regularly decaying density in the sense of Eq. (5), one can connect a given asymptotic behavior of the corresponding potential, e.g., a nonvanishing asymptotic constant or the correct Coulombic $-1 /|\mathbf{r}|$ asymptotic, to the asymptotic enhancement factor $F(s)$ of an exchange-only GGA. The connection is based on an asymptotic differential equation, which was derived in the supplemental material of Ref. [36] and determines $F(s)$ as $s \rightarrow \infty$ for a given asymptotic behavior of the corresponding potential,

$$
F(s)-s\left(1-\frac{1}{2 \ln (s)}\right) F^{\prime}(s)+\frac{1}{4} s^{2} F^{\prime \prime}(s)=v(s) .
$$

The source term on the right-hand side of this equation is uniquely determined by the asymptotic behavior of the 
potential,

$$
v(s) \sim \frac{3}{4 A_{\mathrm{x}}} \frac{v_{\mathrm{x}}^{\mathrm{GGA}}[n, s]}{n^{1 / 3}},
$$

as $s \rightarrow \infty$ via $|\mathbf{r}| \rightarrow \infty$. Therefore, a nonvanishing asymptotic constant corresponds to $v(s) \propto s$, and the correct Coulomb asymptotics corresponds to

$$
v(s)=-\frac{\gamma}{2 A_{\mathrm{x}}} \frac{s}{\ln (s)} .
$$

Thus, up to a linear combination of the two homogeneous solutions $h_{1}(s)$ and $h_{2}(s)$ of this differential equation, $F(s)$ is asymptotically uniquely determined by the behavior of the potential. To leading order, the homogeneous solutions are characterized by the relations

$$
h_{1}(s) \sim s \ln ^{2 / 3}(s)
$$

and

$$
h_{2}(s) \sim \frac{s^{4}}{\ln ^{8 / 3}(s)}
$$

as $s \rightarrow \infty$, i.e., in terms of Eq. (35) by $d=1$ and 4 . Therefore, both homogeneous solutions represent enhancement factors that lead to irregular behavior in the vicinity of nodal surfaces. Consequently, if for a given potential asymptotic the particular solution [79] for $F(s)$ also exhibits nodal surfaces issues, then this specific potential asymptotic is (in the GGA exchange form) incompatible with a potential that behaves regularly in the vicinity of nodal surfaces. This is the case for the criteria of a nonvanishing asymptotic constant and of the correct Coulombic $-1 /|\mathbf{r}|$ asymptotic of the potential, where the asymptotics of the particular solutions are $F(s) \sim B_{1} s \ln (s)$ and $F(s) \sim-\left(\gamma / A_{\mathrm{x}}\right) s$ as $s \rightarrow \infty$, correspondingly. The latter asymptotic is, e.g., used in a recent GGA of CarmonaEspíndola et al. [27].

Moreover, the correct asymptotic behavior of the exchangeenergy density, $e_{\mathrm{x}}(\mathbf{r}) \sim-n(\mathbf{r}) / 2|\mathbf{r}|$, is in the GGA form only realizable by the asymptotic enhancement factor

$$
F(s) \sim-\frac{\gamma}{3 A_{\mathrm{x}}} \frac{s}{\ln (s)},
$$

as implemented in the B88-GGA. Therefore, we conclude based on Sec. IV C that this asymptotic-design criterion is also incompatible with regular behavior close to nodal surfaces.
[1] P. Hohenberg and W. Kohn, Phys. Rev. 136, B864 (1964).

[2] W. Kohn and L. J. Sham, Phys. Rev. 140, A1133 (1965).

[3] J. P. Perdew, R. G. Parr, M. Levy, and J. L. Balduz, Phys. Rev. Lett. 49, 1691 (1982).

[4] M. Mundt and S. Kümmel, Phys. Rev. Lett. 95, 203004 (2005).

[5] M. Lein and S. Kümmel, Phys. Rev. Lett. 94, 143003 (2005).

[6] M. Hellgren and E. K. U. Gross, Phys. Rev. A 85, 022514 (2012).

[7] P. Elliott, J. I. Fuks, A. Rubio, and N. T. Maitra, Phys. Rev. Lett. 109, 266404 (2012).

[8] M. J. P. Hodgson, J. D. Ramsden, and R. W. Godby, Phys. Rev. B 93, 155146 (2016).

[9] T. Dimitrov, H. Appel, J. I. Fuks, and A. Rubio, New J. Phys. 18, 083004 (2016).

[10] D. J. Tozer, J. Chem. Phys. 119, 12697 (2003).

[11] S. Kümmel, L. Kronik, and J. P. Perdew, Phys. Rev. Lett. 93, 213002 (2004).

[12] D. Hofmann and S. Kümmel, Phys. Rev. B 86, 201109 (2012).

[13] F. Wilken and D. Bauer, Phys. Rev. Lett. 97, 203001 (2006).

[14] M. Thiele, E. K. U. Gross, and S. Kümmel, Phys. Rev. Lett. 100, 153004 (2008).

[15] S. R. Whittleton, X. A. S. Vazquez, C. M. Isborn, and E. R. Johnson, J. Chem. Phys. 142, 184106 (2015).

[16] N. L. Nguyen, G. Borghi, A. Ferretti, I. Dabo, and N. Marzari, Phys. Rev. Lett. 114, 166405 (2015).

[17] R. van Leeuwen and E. J. Baerends, Phys. Rev. A 49, 2421 (1994).

[18] A. D. Becke and M. R. Roussel, Phys. Rev. A 39, 3761 (1989).

[19] P. Jemmer and P. J. Knowles, Phys. Rev. A 51, 3571 (1995).

[20] E. Engel and S. H. Vosko, Phys. Rev. B 47, 13164 (1993).

[21] V. N. Staroverov, J. Chem. Phys. 129, 134103 (2008).

[22] R. Armiento, S. Kümmel, and T. Körzdörfer, Phys. Rev. B 77, 165106 (2008).

[23] A. P. Gaiduk and V. N. Staroverov, J. Chem. Phys. 128, 204101 (2008).
[24] A. Karolewski, R. Armiento, and S. Kümmel, Phys. Rev. A 88, 052519 (2013).

[25] F. Tran, P. Blaha, and K. Schwarz, J. Chem. Theory Comput. 11, 4717 (2015).

[26] F. Tran, P. Blaha, M. Betzinger, and S. Blügel, Phys. Rev. B 94 , 165149 (2016).

[27] J. Carmona-Espíndola, J. L. Gázquez, A. Vela, and S. B. Trickey, J. Chem. Phys. 142, 054105 (2015).

[28] A. D. Becke and E. R. Johnson, J. Chem. Phys. 124, 221101 (2006).

[29] F. Tran and P. Blaha, Phys. Rev. Lett. 102, 226401 (2009).

[30] E. Räsänen, S. Pittalis, and C. R. Proetto, J. Chem. Phys. 132, 044112 (2010).

[31] D. Koller, F. Tran, and P. Blaha, Phys. Rev. B 85, 155109 (2012).

[32] F. Tran, P. Blaha, and K. Schwarz, J. Phys.: Condens. Matter 19 196208 (2007).

[33] D. J. Singh, Phys. Rev. B 82, 205102 (2010).

[34] D. Koller, F. Tran, and P. Blaha, Phys. Rev. B 83, 195134 (2011).

[35] F. Tran, P. Blaha, M. Betzinger, and S. Blügel, Phys. Rev. B 91, 165121 (2015).

[36] R. Armiento and S. Kümmel, Phys. Rev. Lett. 111, 036402 (2013).

[37] T. Aschebrock, R. Armiento, and S. Kümmel (unpublished).

[38] F. Della Sala and A. Görling, J. Chem. Phys. 116, 5374 (2002).

[39] F. Della Sala and A. Görling, Phys. Rev. Lett. 89, 033003 (2002).

[40] S. Kümmel and J. P. Perdew, Phys. Rev. Lett. 90, 043004 (2003).

[41] S. Kümmel and J. P. Perdew, Phys. Rev. B 68, 035103 (2003).

[42] M. Mundt, S. Kümmel, B. Huber, and M. Moseler, Phys. Rev. B 73, 205407 (2006).

[43] L. Kronik, A. Makmal, M. L. Tiago, M. M. G. Alemany, M. Jain, X. Huang, Y. Saad, and J. R. Chelikowsky, Phys. Status Solidi B 243, 1063 (2006).

[44] A. Makmal, S. Kümmel, and L. Kronik, J. Chem. Theory Comput. 5, 1731 (2009). 
[45] A. Makmal, S. Kümmel, and L. Kronik, J. Chem. Theory Comput. 7, 2665 (2011).

[46] S. Kümmel and L. Kronik, Rev. Mod. Phys. 80, 3 (2008).

[47] Q. Wu, P. W. Ayers, and W. Yang, J. Chem. Phys. 119, 2978 (2003).

[48] P. Gori-Giorgi, T. Gál, and E. J. Baerends, Mol. Phys. 114, 1086 (2016).

[49] T. Schmidt, E. Kraisler, L. Kronik, and S. Kümmel, Phys. Chem. Chem. Phys. 16, 14357 (2014).

[50] One can argue that Ref. [58] shows that the ground-state density of a fermionic many-electron system can be mapped to the ground state of a bosonic system if one assumes that the effective potential of Ref. [58] is regular and does not introduce nodes. Under this condition, one can conclude that fermionic ground-state densities are nodeless due to fact that the ground-state wave function of any bosonic system is nodeless [80].

[51] A. D. Becke, Phys. Rev. A 38, 3098 (1988).

[52] A. D. Becke, J. Chem. Phys. 98, 5648 (1993).

[53] F. J. Devlin, J. W. Finley, P. J. Stephens, and M. J. Frisch, J. Phys. Chem. 99, 16883 (1995).

[54] W. E. Pickett, Comput. Phys. Rep. 9, 115 (1989).

[55] J. R. Chelikowsky and M. L. Cohen, Handbook on Semiconductors (Elsevier, Amsterdam, 1992), p. 59.

[56] J. B. Krieger, Y. Li, and G. J. Iafrate, Phys. Rev. A 46, 5453 (1992).

[57] Wolfram Research, Inc., MATHEMATICA 8.0 (2010).

[58] M. Levy, J. P. Perdew, and V. Sahni, Phys. Rev. A 30, 2745 (1984).

[59] J. Jaramillo, G. E. Scuseria, and M. Ernzerhof, J. Chem. Phys. 118, 1068 (2003).

[60] J. C. Slater, Phys. Rev. 81, 385 (1951).

[61] A. P. Gaiduk and V. N. Staroverov, J. Chem. Phys. 131, 044107 (2009).

[62] A. Karolewski, R. Armiento, and S. Kümmel, J. Chem. Theory Comput. 5, 712 (2009).
[63] M. Mundt, S. Kümmel, R. van Leeuwen, and P.-G. Reinhard, Phys. Rev. A 75, 050501(R) (2007).

[64] S. L. Li and D. G. Truhlar, J. Chem. Theory Comput. 11, 3123 (2015).

[65] E. Engel, J. A. Chevary, L. D. Macdonald, and S. H. Vosko, Z. Phys. D 23, 7 (1992).

[66] R. Armiento and A. E. Mattsson, Phys. Rev. B 72, 085108 (2005).

[67] A. P. Gaiduk and V. N. Staroverov, J. Chem. Phys. 136, 064116 (2012).

[68] A. Lembarki, F. Rogemond, and H. Chermette, Phys. Rev. A 52, 3704 (1995).

[69] J. P. Perdew and Y. Wang, Phys. Rev. B 33, 8800(R) (1986).

[70] A. D. Becke, J. Chem. Phys. 85, 7184 (1986).

[71] L. Vitos, B. Johansson, J. Kollár, and H. L. Skriver, Phys. Rev. B 62, 10046 (2000).

[72] M. Levy, Phys. Rev. A 43, 4637 (1991).

[73] L. Pollack and J. P. Perdew, J. Phys.: Condens. Matter 12, 1239 (2000).

[74] J. P. Perdew, A. Ruzsinszky, J. Sun, and K. Burke, J. Chem. Phys. 140, 18A533 (2014).

[75] A. D. Becke, J. Chem. Phys. 84, 4524 (1986).

[76] J. P. Perdew, K. Burke, and M. Ernzerhof, Phys. Rev. Lett. 77, 3865 (1996).

[77] M. Filatov and W. Thiel, Phys. Rev. A 57, 189 (1998).

[78] T. F. T. Cerqueira, M. J. T. Oliveira, and M. A. L. Marques, J. Chem. Theory Comput. 10, 5625 (2014).

[79] Our reasoning here is that we are always looking at such particular solutions that are not asymptotically equivalent to either $h_{1}$ or $h_{2}$. This can always be achieved because due to the linearity of the differential equation, homogenous contributions can be subtracted.

[80] R. P. Feynman, Statistical Mechanics: A Set of Lectures (Addison-Wesley, New York, 1972), pp. 321-323. 